\title{
An evaluation of different IP traceback approaches
}

\author{
Vadim Kuznetsov, Andrei Simkin, and Helena Sandström \\ Department of Computer Science and Electrical Engineering \\ Luleå University of Technology, SE-971 87 Luleå, Sweden
}

\begin{abstract}
The problem of identifying the sources of a denial of service attack is among the hardest in the Internet security area, especially since attackers often use incorrect, or spoofed, source IP addresses. In this paper we present the results from a comparison between some of the most promising traceback techniques proposed to solve this problem. Our goal was to evaluate and analyse the most promising techniques on our way to find a more efficient approach. We have evaluated four different traceback approaches and summarized the results. Our own research were primary targeted at the iTrace approaches while the other approaches were evaluated based on the previous work. We conclude that there are two main disadvantages of the proposed approaches. First, the hop-byhop path reconstruction is inefficient due to a significant computation overhead, or a long time spent for collecting the samples of the path. Second, the path reconstruction requires changes in the core routing structure that is not profitable. We also suggest a slightly modified version of iTrace approach which aims at reducing the overhead imposed by such changes.
\end{abstract}

\section{Introduction}

Denial-of-service (DOS) attacks are a pressing problem in today's Internet. Their impact is often more serious than network congestion due to their targeted and concentrated nature. In a distributed DOS (DDOS) attack, the attacker uses a number of compromised slaves to increase the transmission power and orchestrate a coordinated flooding attack. Highly automated attack tools have been developed where a common ingredient is the use of spoofed source addresses. Particularly, DDOS attacks with hundreds or thousands of compromised hosts, often residing on different networks, may lead to the target system overload and crash. Due to the stateless nature of the Internet, the dilution of locality in the flooding stream combined with spoofed source addresses undermines the effectiveness of traceback techniques for locating the sources. By the use of IP spoofing, stepping stone techniques, and zombie slaves, attackers can quite easily hide their identity. Therefore, finding the true identity of an attacker includes many steps of which tracing the machines that directly generates the attack packets really is only the first step.

Another way to render efficient DDOS attacks, which do not include the use of compromised slaves, is by bounc- ing flooding traffic off of reflectors. Attackers can thereby effectively hide their own location. In this scenario the attacker sends a false request to the reflector on behalf of the victim, which is done by setting the victim IP address as the source address. By spoofing requests from the victim to a large set of Internet servers (for example DNS, Gnutella, and web servers) attackers can make it very difficult for a victim to isolate the attack traffic in order to block it. As pointed out in [11] it proves impractical to defeat reflector attacks with traceback techniques, especially since the source addresses that reach a victim are the true IP address of each reflector. Therefore reflector attacks are not particularly considered in the context of traceback.

During recent years the problem with DOS attacks has become well known, but it has been difficult to find a way to measure the denial of service activity in the Internet as a whole. As far as we know there is only one publication in this area [4]. In this publication they have been able to measure, with some reliability, the activity of DOS attacks (excluding reflector attacks). The experimental measurements give a conservative lower bound of approximately 20-40 attacks per hour in the whole Internet, directed to different network prefixes, with the mean duration of 1015 minutes. If these results are correct, they give us a very sad imagination of today's Internet security.

The problem of tracing streams of spoofed packets has received considerable attention recently, and several approaches have been introduced in the Internet society. One technique is Ingress filtering as described in RFC 2827 [13]. The idea with ingress filtering is that packets from an edge network should be filtered using the prefix for that network. Any packet carrying an IP address with a wrong prefix should be blocked at the filtering router and not allowed to continue towards its destination. If ingress filtering could be implemented everywhere tracing traffic would be unnecessary. The problem is that it is not likely that all edge networks will ever implement this even though it is in use today. Therefore our believe is that it will still be interesting to find efficient traceback techniques.

The rest of this paper is organized as follows. We provide a short description of proposed techniques in Section 2. In Section 3 we introduce the parameters we have used for comparison. In Section 4 we present a modified iTrace approach and describe result in Section 5. Finally we summarize in Section 6, and conclude in Section 7. 


\section{Traceback approaches}

We have chosen to sort proposed traceback techniques into three distinct categories:

1. Actively querying routers about traffic they forward.

2. Creating a virtually overlay network for selective monitoring of packet flows.

3. Identifying the attack path by reconstruction, using a collection of packets, marked or especially generated by routers along the attack path.

A simple scheme of the first category is in use today. If a victim recognizes that it is being attacked it develops an attack signature, consisting of some data common and unique to the attack traffic. A query including the attack signature is then sent hop-by-hop to each router along the path. This presuppose that each routing device supports input debugging and is able to tell through which interface a packet corresponding to the attack signature arrived. This technique is however not very efficient and requires a lot of manpower and good contacts with other network providers. Some ISP's ${ }^{1}$ may have implemented a more sophisticated and automated technique for this to speed up the trace procedure within their own network. A drawback of all techniques in the first category is that tracing can only be done during an ongoing attack.

In the second category we have placed different logging techniques $[8,12]$. In general it is not feasible to use logging since it often requires huge storage capacities, but within this category there is one very interesting approach, Hash-Based IP traceback [8], more common called SPIE. With the use of an efficient logging technique, only collecting hashes of the packets, it is possible to trace a single route of one packet.

The third category includes different variants of probabilistic packet marking (PPM), first proposed by Savage and colleagues [2], and ICMP traceback (iTrace), first proposed by Bellovin and colleagues [5].

The basic idea behind PPM is the use of edge sampling. A packet on the path is with a certain probability marked by two routers on the way, forming an edge. Each marked packet then represents a sample of the whole path. The victim receives all packets and can thereby use the marked packet to reconstruct the entire path back to the source. An enhanced version of PPM has been presented by Song and Perrig [3]. They noticed that if the victim knows the map of its upstream routers the computation and reconstruction can be done much more efficient.

The basic idea behind iTrace is that every router should sample a packet with some probability, copy its content onto a special ICMP packet, add information about the adjacent upstream and/or downstream routers and send it towards the same destination as the original packet. The victim of an attack can then use these packets to reconstruct

\footnotetext{
${ }^{1}$ Internet Service Providers
}

the paths back to the attackers. An observation made of this solution was that it is much more likely that the victim will get iTrace packets from routers nearby than from routers far away. A variant of iTrace, called intention-driven iTrace $[6,7]$ propose a solution to this, which increase the probability of receiving an iTrace message when needed. By using a special intention value that can be propagated to routers through $\mathrm{BGP}^{2}$ updates it is possible for a host or victim to raise the probability of receiving iTrace packets from remote routers.

A common disadvantage of the first category is that tracing can not be done post mortem, after an attack has stopped. Within the second category, all techniques expect SPIE requires huge storage capacity. Even though SPIE is a promising technique, and is unique in that it can trace a single packet, it suffers from a very tight timelimit and costly investments. Therefore we have chosen to focus on the various techniques proposed within the third category.

\section{Evaluation of the suggested ap- proaches}

We have simulated the different traceback approaches on a linear, star, and tree topology. The results of the simulations were verified and compared to the results of mathematical analysis, where possible.

We have selected four distinct parameters for comparison and evaluation of different traceback approaches,

1. the number of packets required for a complete path reconstruction

2. the computation overhead of a reconstruction procedure

3. the robustness of a traceback mechanism in case of a large-scale DDOS

4. the deployment overhead

The first parameter represents estimated time for collecting enough packets to get required information for the reconstruction. The second parameter represents estimated time required for processing collected information to be able to reconstruct the path back to the attackers. It is desirable to minimize these two characteristics to achieve a fast response to an attack, and diminish the damage. The third parameter, the definition of robustness, was first given by Song and Perrig [3]. They introduced two terms: false positive, a path that do not take part in an attack but it is reconstructed by a traceback mechanism, and false negative, a path that take part in an attack but it is not reconstructed. The traceback approach is robust if it gives a relatively low rate of false positives and false negatives, and if the rate does not grow rapidly with increased number of attackers.

\footnotetext{
${ }^{2}$ Border Gateway Protocol, an interdomain routing protocol by which autonomous systems (groups of networks and routers) exchange reachability information.
} 


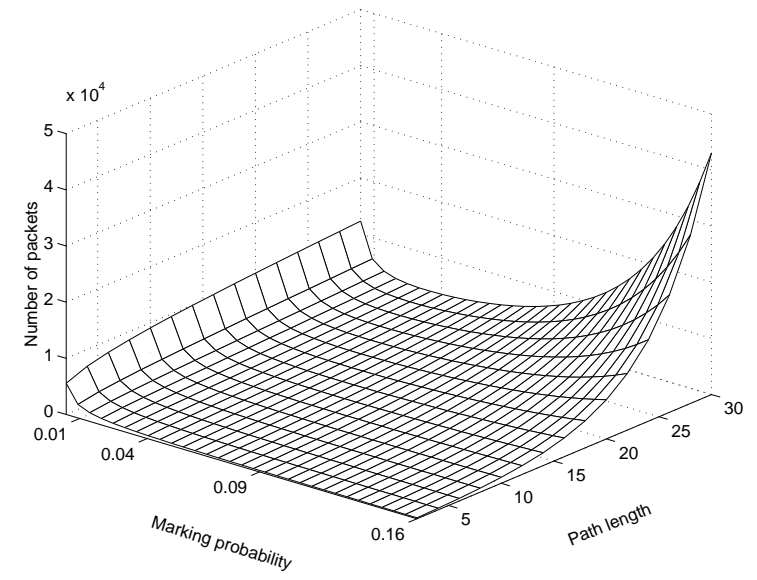

Figure 1: The expected number of packets required for path reconstruction in Savage and colleagues algorithm

The fourth parameter, the deployment overhead, can not be measured or calculated directly but can be evaluated by means of common sense.

The choice of these parameters is motivated by our aspiration for a solution to the IP traceback problem that is time and cost efficient, and gives a high precision.

\subsection{Methodology}

Our evaluation is based on the contribution of previously published papers $[2,3,7]$, simulations in the network simulator [10] and mathematical analysis.

The network simulator was extended to be used with both the PPM methods as well as with the two approaches of iTrace. The attack traffic was simulated by the UDP agents attached to every attack node and which were configured to send traffic with a rate of about $500 \mathrm{pkt} / \mathrm{s}$. The connecting links between routers were configured to propagate data with high speed and minimal loss-rate in the queues (this was achieved by setting up high bandwidth of the links ). A victim node collected all required data and stored it into a file. These data were further processed in matlab. The goal of the simulations was to evaluate statistically the behavior of the chosen approaches therefore every simulation with the same configuration was performed 100 times. In our simulation we used different topologies: linear topology, star and tree topology. In case of a tree topology a victim was located at the root of a balanced tree while the attackers were at the leaves of the tree.

\subsection{Number of packets required for recon- struction}

The purpose of path reconstruction is to find the IP address of an attacking host or at least the address of the router closest to the attacking host. During the reconstruction procedure we have to verify that the reconstruction path is complete and correct. Neither PPM nor iTrace approaches provide a mechanism for verifying the completeness of the

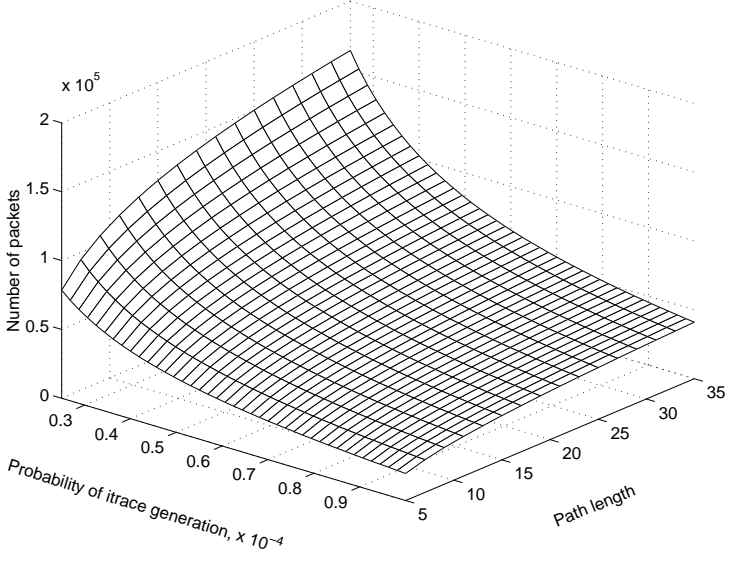

Figure 2: The expected number of attacking packets required for path reconstruction in iTrace

reconstructed path. The only way to verify this is to collect accordingly large amount of samples, and to make sure that there are no new samples.

The edge marking algorithm in PPM depends primarily on the marking probability $p$. This parameter defines the fraction of packets which are marked by a router. The expected number of collected packets required for path reconstruction for a linear topology is bounded by:

$$
\mathbf{E}\{N\}=\frac{k \cdot \ln (k \cdot d)}{p(1-p)^{d-1}}
$$

where $k$ is the number of samples required for reconstruction of a single edge between two routers and $d$ is the length of the path from the victim to the attacker [2].

The method suggested by Savage and colleagues assumes $k=8$. Figure 1 represents the graph of this equation

As Figure 1 indicates, with a value of $p$ around 4-5\%, the number of packets required for path reconstruction is minimized, and does not exceed $4 \cdot 10^{3}$ for all values of $d$ in the interval between 2 and 32. It is remarkable that this number neither depends on topology nor the number of attackers. Assuming that the rate of emitting attack packets is equal to 10 packets per second, per attacking host (which is an extremely low rate [4]), we yield that the attacking hosts have to generate traffic as long as 5-7 minutes before a victim is able to reveal the whole path.

The method proposed by Song and Perrig requires less packets although the robustness suffers in this case [3]. The iTrace approach depends on the ICMP packet generating probability $\mu$, which indicates the fraction of the packets being traced by a particular router. If, for example, this parameter is equal to $1 / 20000$, it means that one packet out of 20000 is picked up to be traced and an ICMP packet containing information about the route of this packet is issued towards the destination. Given that routers add one adjacent router, the reconstruction of the path from a victim to an attacker is possible if all the routers have generated at least one ICMP packet. We have found an appropriate formula showing the expected number of packets required to 
be generated by an attacking host (in case of linear topology), in order to make sure that all routers along the path have generated at least one ICMP packet.

$$
\mathbf{E}\{N\}=\frac{\ln (d-1)+0.58}{\mu}
$$

where $d$ is the length of the path from the victim to the attacking host. The proof of the formula is given in the Appendix. Figure 2 represents the graph of this equation.

Figures 3 and 4 show the dependency on a distance from an attacker to a victim for the particular values of parameters $p$ (for Savage and colleagues) and $\mu$ (for iTrace) correspondingly. These figures shows also a good correlation of the theoretical and simulated results.

For the proposed value of $\mu=1 / 20000$ and the mean Internet diameter 20 hops the whole path can be reconstructed after an attacking host emits about $10^{4}-10^{5}$ packets. However, in real life, this value should be at least 10 times higher. This correction is introduced by the following two reasons. First, the simulations on the network simulator have shown the dependency on the routing topology. Figure 5 represents the graph of the expected number of packets in case of a binary tree topology and 3-ary tree topology compared to a linear topology. This figure shows that the number of packets required for path reconstruction may be significantly higher in case of a complicated topology even if only the attack traffic presents.

Second, the rate of attack packets emitted by a particular host can be quite low compared to the legitimate background Internet traffic. This reduces the probability that an attack packet can be sampled by a router. Assuming an average rate of about $10^{2}$ emitting attack packets per second per attacking host [4], we yield that the attacking hosts should generate traffic as long as 2-3 hours before a victim is able to reveal the whole path. The parts of the path, however, can be reconstructed after a few minutes, but the pieces of the path, obviously, do not disclose the origin of the attack, although they can provide valuable information pointing where to add traffic filters. In order to minimize the total number of attack packets required for path reconstruction, we have to raise the generating probability $\mu$ at least 10-20 times. However, this could be inappropriate due to the significant traffic overhead caused by the iTrace packets. Intention-driven iTrace can improve the situation and eliminate the influence of the topology and background traffic, although the propagation of the "intention" value through BGP updates may be quite slow, delaying the invocation of the intention driven mechanism. Another problem with intention driven iTrace is that it may cause instability in the routing mechanism due to frequent updates in the routing table.

\subsection{Computation overhead}

PPM, as suggested by Savage and colleagues, suffers from enormous computation overhead in case of a largescale DDOS. The complexity of reconstruction grows very

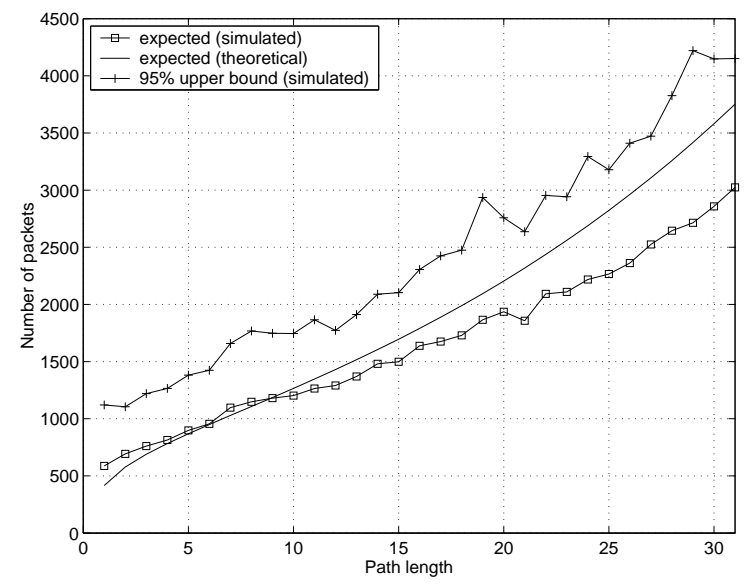

Figure 3: The expected number of packets required for path reconstruction in Savage and colleagues algorithm in case of a linear topology $(p=0.04)$

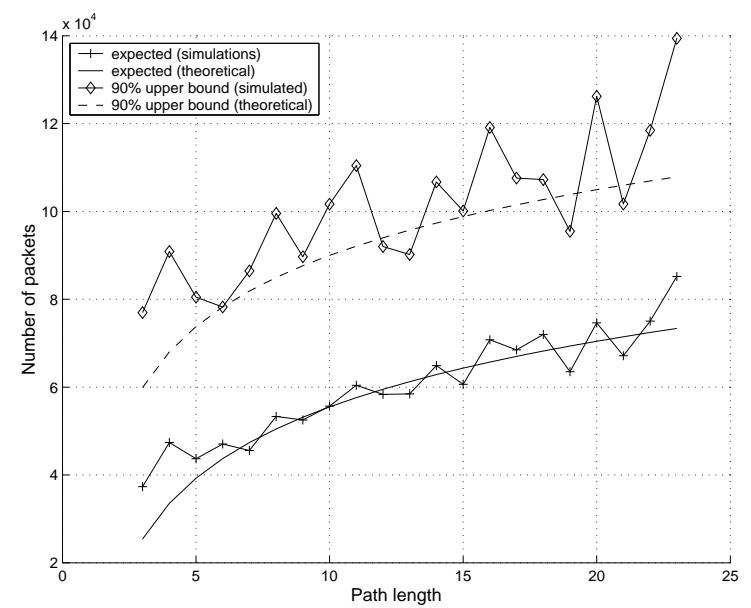

Figure 4: The expected number of attacking packets required for path reconstruction in iTrace in case of a linear topology $\left(\mu=1 / 2^{15}\right)$

rapidly and is upper bounded by $O\left(l \cdot n^{8}\right)$, where $l$ is the maximal distance to the attackers and $n$ is the number of attacking hosts. The large overhead is explained by the constraint to break the IP address of a router into eight pieces to fit each piece into the fragmentation field in the IP header. Consequently, the path reconstruction of all attacking sources can take hours or days [3].

The complexity of the path reconstruction in the traceback approach proposed by Song and Perrig depends primarily on the topology of the map of upstream routers instead of the number of attackers, and does not have a large overhead. The complexity of the path reconstruction is upper bounded by $O(l \cdot n)$ and therefore scales well, although the reconstruction procedure requires some intensive calculations. The complexity of the reconstruction procedure in iTrace is upper bounded by $O(l \cdot n)$, and the reconstruction mechanism does not require significant and cumbersome calculations. 


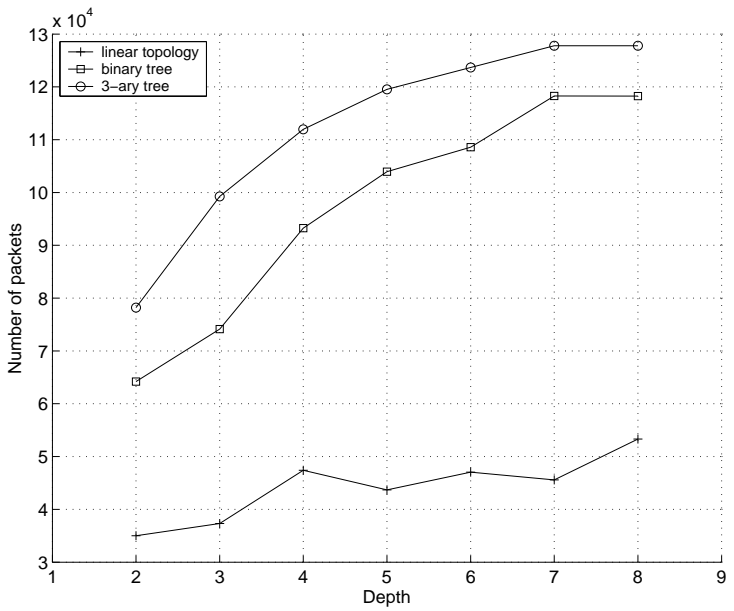

Figure 5: The expected number of attacking packets per attacking host required for path reconstruction in iTrace in case of a linear and tree topology $\left(\mu=1 / 2^{15}\right)$

\subsection{Robustness}

In terms of robustness both the PPM approaches are insufficiently robust. The Savage algorithm gives a very high rate of false positives. Simulations in the network simulator have shown that false positives can even appear when the number of attackers is as low as five. Such behavior is explained, again, by a constraint to break the router's IP address into eight pieces, rising the probability of the accidentally incorrect reconstructed paths at the victim side. The Song and Perrig approach instead suffers from a possible high rate of false negatives. This is primary connected to the use of a map of upstream routers. If the map is not accurate or not correspond to the real topology, then the paths to some attackers cannot be reconstructed. In fact, no PPM approach is "collision-resistant" in the sense that ambiguous representation of the same edge increases the rate of false positives during the reconstruction procedure. The iTrace approach introduces a higher robustness, and does not suffer much from false positives. However, a host may not be discovered if it is sending a low volume of attack traffic. The robustness of iTrace is more influenced by insufficient information about the attack paths gathered at the victim, rather than ambiguous representation of information.

We would like to note that any traceback approach, which uses a path reconstruction mechanism, will suffer from false positives due to two main reasons. First, it is difficult to prove whether the path is reconstructed completely or partly. A partially reconstructed path is considered as a false negative, since the reconstruction procedure returns the IP address of a host which is not actually involved in the attack and just lies somewhere in the middle of the path. Second, a single mistake on one step of the hop-by-hop path reconstruction procedure can imply multiple false negatives on the subsequent steps.

\subsection{Deployment overhead and cost}

One of the desired features of a traceback approach is incremental deployment into the current Internet structure, at low cost. The expenses for deployment of traceback should not exceed the losses from the denial of service activity. Unfortunately, all proposed approaches require a significantly high level of expenses since they require changes in routers present in the current Internet structure. The authors of the PPM scheme claim that their approach is incrementally deployable but this has not been thoroughly evaluated. For iTrace, the reconstruction of the whole path requires the participation of every other router on the path. Intention-driven iTrace has the drawback that it imposes a change in a somewhat sensitive BGP protocol.

\section{A modified iTrace approach}

As we have seen from the previous sections, the main drawbacks of the iTrace approach are a large number of attacking packets required for the path reconstruction and significant overhead caused by necessity to implement the iTrace mechanism on every router. While the first drawback can be (at least, theoretically) solved by tuning the generating probability $\mu$, the second drawback requires a modification in the mechanism itself in order to add such a desirable characteristics as "incremental deployment" and reduce the overhead by implementing the iTrace mechanism only an a small amount of the routers.

An obvious improvement of the second drawback is the marking of the iTrace messages (issued by one router) on the other routers thereby creating a chain of the routers which the message traverses on its path. However, this method suppose checking every packet in a router, which is inefficient. Instead, we suggest to select packets with a relatively low probability (which we denote as a selecting probability $\eta$ ) from the queue in a router and if the selected packet is an iTrace message then mark it by adding some additional information to the body of the message. In other words, the router that implements iTrace should not only generate iTrace messages but also examine the randomly chosen packets and, if the examined packet is an iTrace message from another router, add its own IP address to a special field of the iTrace message. The reconstruction procedure at the victim side will use this information to fill in the "gaps" between the routers which do not implement the iTrace mechanism.

The selecting probability $\eta$ should be low to avoid a processing overhead but, from the other hand, it should not be very low, since this decreases the probability of marking iTrace messages by the other routers. We suggest to select the value for $\eta$ from 0.05 to 0.1 , because this gives an insignificant processing overhead on a router and, as experiments have shown, this value is sufficient for marking a reasonably high amount of iTrace messages.

We have proved that this approach allows successfully reconstruct the path back to the router which is the nearest 


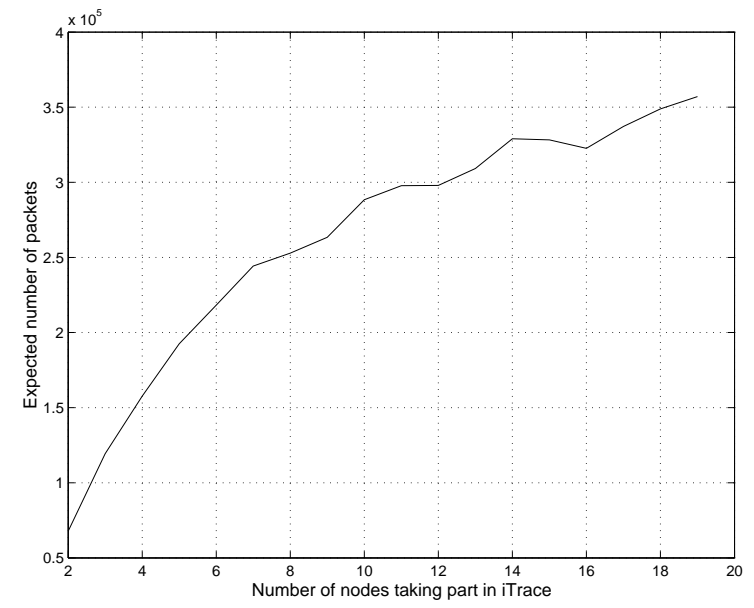

Figure 6: The expected number of attacking packets required for path reconstruction in a modified iTrace approach in case of a linear topology $\left(\mu=1 / 2^{15}, \eta=\right.$ $1 / 2^{4}$ )

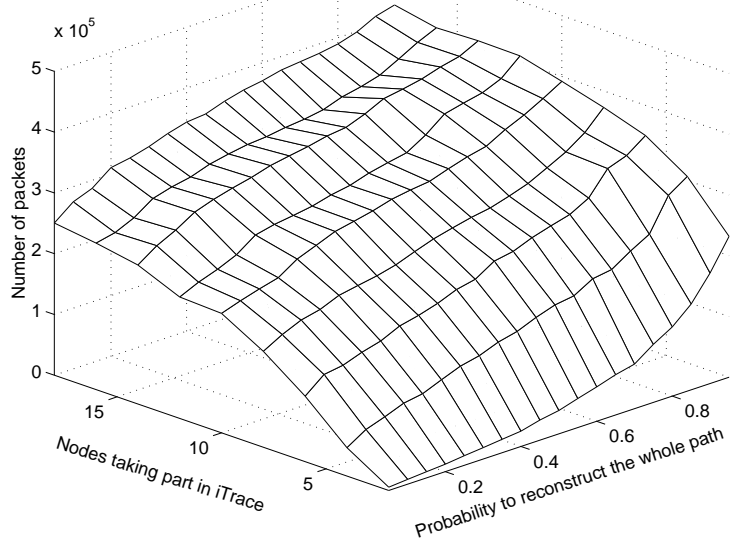

Figure 7: The number of attacking packets required for reconstruction of the whole path with the given probability in case of a linear topology $\left(\mu=1 / 2^{15}, \eta=1 / 2^{4}\right)$

to the attaching host and which implements the modified iTrace mechanism. The reconstruction is possible even if only a part of the routers implements the modified iTrace mechanism. Moreover, a small amount of such routers, which located in some distance from each other, can do this work better than a large amount of routers disposed in a raw.

The following simulations were conducted in the network simulator. We have connected an attacking node, a "gap", a chain of the nodes which implement the modified iTrace mechanism and a victim into a linear topology. The "gap" was represented by several nodes which do not implement an iTrace mechanism at all. The length of the chain of the nodes which implement the modified iTrace mechanism varied from two up to twenty. The reconstruction procedure was extended to cope with the iTrace messages marked by the other nodes on its way. The purpose of these simulations was to figure out how the chain of nodes influences to the path reconstruction.

The result of the simulations is shown in Figures 6 and 7. Figure 6 indicates the dependency of the expected number of attacking packets required for path reconstruction on the length of the chain. Figure 7 is a more extended analysis of our result. It shows the number of packets, which an attacking host should emit in order to reconstruct the whole path with a certain probability given in advance.

As the figures indicate, it is not desirable to have a long chain of routers which implements a modified iTrace approach because this complicates the reconstruction and requires more packets to complete the reconstruction. This phenomena is explained as follows. As we mentioned above in Section 3.2, the reconstruction procedure makes a conclusion that the path is reconstructed completely if it receives no new samples of the path. The longer chain requires more packets for its reconstruction and emits more new samples of the path thereby delaying the reconstruction procedure with a conclusion that the reconstruction is complete.

\section{Result}

The desired characteristics of an IP traceback mechanism are that it requires a relatively small number of packets for path reconstruction, low complexity of reconstruction, high robustness, and low deployment overhead and cost. We consider all parameters equally important since a low evaluation of one of the parameters opposes using the method in practice.

Table 1 indicates that none of the proposed solutions satisfies all the desired parameters. Both PPM approaches have insufficient robustness in case of a DDOS attack but require fewer packets than iTrace for path reconstruction, whereas both of the iTrace approaches possess a relatively good robustness. The deployment overhead is high for all the methods (except the modified iTrace approach) since all of them assume some kind of alternation in routers in the Internet. The modified iTrace approach assumes alternation only in a small and carefully selected part of routers therefore the deployment overhead is less in this case. Summarizing the above, we infer that each of the suggested approaches is directed to solve one particular issue of the IP traceback problem but none can solve all of them.

\section{Summary}

In this paper we have closely studied and evaluated four IP traceback approaches proposed during the last years. We have identified four parameters which can be used for the comparison. These parameters are aimed at finding a time and cost efficient approach with high precision. Evaluation of the edge marking approach was based on previously published articles and on our own simulations, while 


\begin{tabular}{|c|c|c|c|c|}
\hline & $\begin{array}{l}\frac{n}{0} \\
\frac{u}{0} \\
\tilde{\tilde{n}}\end{array}$ & 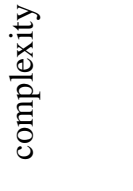 & $\begin{array}{l}\mathscr{n} \\
0 \\
0 \\
\Xi \\
0 \\
0 \\
0\end{array}$ & 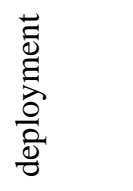 \\
\hline $\begin{array}{l}\text { Savage et } \\
\& \text { colleges }\end{array}$ & $4 \cdot 10^{3}$ & $O\left(l \cdot n^{8}\right)$ & low & high \\
\hline $\begin{array}{ll}\text { Song } & \& \\
\text { Perrig } & \end{array}$ & $<4 \cdot 10^{3}$ & $O(l \cdot n)$ & medium & high \\
\hline iTrace & $>10^{6}$ & $O(l \cdot n)$ & high & high \\
\hline $\begin{array}{l}\text { intention } \\
\text { driven } \\
\text { iTrace }\end{array}$ & $10^{5}$ & $O(l \cdot n)$ & high & $\begin{array}{l}\text { very } \\
\text { high }\end{array}$ \\
\hline $\begin{array}{l}\text { modified } \\
\text { iTrace }\end{array}$ & $\sim 10^{6}$ & $O(l \cdot n)$ & high & medium \\
\hline
\end{tabular}

Table 1: Summary of IP traceback approaches evaluation

the evaluation of the iTrace mechanism was based on our own research and simulations in the network simulator. We have also suggested a modified iTrace approach which aims at reducing the deployment overhead.

\section{Conclusion and discussion}

The detailed analysis have revealed two main disadvantages of the previously proposed approaches. First, the hop-by-hop path reconstruction is inefficient due to a significant computation overhead, or a long time spent for collecting the samples of the path. Second, the path reconstruction imposes changes in the core routing structure that is not profitable. With this in mind, a conclusion is that it may be a better approach to solve the IP traceback problem locally, concentrating on the first hop router, or the router that connects a local network to the rest of the Internet. The goal of all reconstruction algorithms is to find the sources of the attacking traffic, but the reconstruction of an attack path can actually only reveal the first router an attack packet has passed. Since the first router is of main interest, it would be desirable to find an algorithm that could reveal the identity of the first router, without requiring the participation of all the routers on the path. However, it is not practically to rely only on the first-hop router because it may be compromised or damaged. The better solution would be to include a traceback support into the core Internet structure itself but to limit the overhead imposed by such changes. The modified iTrace approach that we have presented in this article is an example of such a solution. Our future research will focus on finding improvements in this approach.

\section{References}

[1] S. C. Lee and C. Shields, "Tracing the Source of Network Attack: A Technical, Legal and Societal Problem", Proc. of the 2001 IEEE Workshop on Information Assurance and Security, June 2001, United States Military Academy, West Point, NY, pp 239-246,http://www.research.att.com/ lists/ietf-itrace/2000/09/msg00044.html

[2] Stefan Savage, David Wetherall, Anna Karlin and Tom Anderson, "Practical Network Support for IP Traceback", Proc. of the ACM SIGCOMM conference, August 2000, Stockholm, Sweden, Computer Communication Review Vol. 30, No 4, October 2000, pp. 295-306,www.cs.washington.edu/homes/ savage/papers/Sigcomm00.ps

[3] Dawn X. Song and Adrian Perrig, "Advanced and Authenticated Marking Schemes for IP Traceback", Proc. of the IEEE infocom conference, april 2001, Anchorage, Alaska,http://paris.cs.berkeley.edu/ perrig/ projects/iptraceback/tr-iptrace.pdf

[4] David Moore, Geoffrey M. Voelker and Stefan Savage, "Inferring Internet Denial-of-Service Activity", Proc of the 10th USENIX Security Symposium, August 2001, Washington D.C.,http://www.caida.org/outreach/papers/ 2001/BackScatter/usenixsecurity01.pdf

[5] Steven M. Bellovin, "ICMP Traceback Messages", Internet Draft: draft-bellovin-itrace-00.txt, submitted Mar. 2000, expiration date Sep. 2000,http://www.research.att.com/ $\sim$ smb/papers/draft-bellovin-itrace-00.txt

[6] S. Felix Wu, Lixia Zhang, Dan Massey, Allison Mankin, "Intention-Driven ICMP Trace-back", Internet Draft: draft-wuitrace-intention-00.txt, submission date Feb. 2001, expiration date Aug. 2001,http://www.ietf.org/internet-drafts/ draft-ietf-itrace-intention-00.txt

[7] Allison Mankin, Dan Massey, Chien-Long Wu, S. Felix Wu, Lixia Zhang, "On Design and Evaluation of IntentionDriven ICMP Traceback", Proc. of 10th IEEE International Conference on Computer Communications and Networks, Oct. 2001, Scotsdale Arizona,http://irl.cs.ucla.edu/ papers/Intention-iTrace.pdf

[8] Alex C. Snoeren et al., "Hash-Based IP Traceback", Proc. of the ACM SIGCOMM conference 2001, San Diego, CA, Computer Communication Review Vol. 31, No 4, October 2001, pp. 3-14,http://nms.lcs.mit.edu/ snoeren/ papers/spie-sigcomm.pdf

[9] Drew Dean, Matt Franklin, Adam Stubblefield, "An Algebraic Approach to IP Traceback", Xerox PARC, Rice University, August 2000,http://www.cs.rice.edu/ astubble/ algtrace.pdf

[10] The Network Simulator - ns-2 http://www.isi.edu/ nsnam/ns

[11] Vern Paxson, "An Analysis of Using Reflectors for Distributed Denial-of-Service Attacks", ACM SIGCOMM Computer Communication Review Vol. 31, No 3, July 2001,http://www.acm.org/sigcomm/ccr/archive/ 2001/jul01/ccr-200107-paxson.pdf

[12] Robert Stone "CenterTrack: An IP Overlay Network for Tracking DoS Floods", Proceedings of the 9th USENIX Security Symposium, Aug 2000,http://www.usenix.org/ publications/library/proceedings/sec2000/ full_\{p\}apers/stone/stone.pdf

[13] P. Fergusson and D.Seine, "Network Ingress Filtering: Defeating Denial of Service Attacks which employ IP Source Address Spoofing", RFC2827, May 2000,http://www.ietf.org/rfc/ rfc2827.txt 


\section{Appendix}

\section{The expected number of packets re- quired for path reconstruction in iTrace approach}

Let us consider a trivial linear topology with a victim $d$ hops away from an attacker. There are $d-1$ routers between the attacker and the victim. Let event $\mathbf{A}_{i}$ be the event where a router $R_{i}$ emits at least one iTrace packet.

$$
\mathbf{A}_{i}, i=\overline{1, d-1}
$$

The probability of such an event depends on probability of traceback generating probability $\mu$ and number of packets $N$ sent by the attacker

$$
\operatorname{Pr}\left\{\mathbf{A}_{i}\right\}=1-(1-\mu)^{N} \approx 1-e^{-N \mu}
$$

Consider for the beginning that the reconstruction of the reverce path from the victim to the attacker is based only on backward links, i.e, we assume that iTrace packets include only backward links. In this case the reconstruction is only possible if all routers emits at least one iTrace packet. Let event $\mathbf{A}^{\mathbf{d}}$ be an event where all routers between the victim and the attacker emits at least one iTrace packet after $N$ packets are gone through the path.

$$
\mathbf{A}^{\mathbf{d}}=\bigcap_{i=1}^{d-1} \mathbf{A}_{i}
$$

Since the probability of event $\mathbf{A}^{\mathbf{d}}$ plays further an important role we define the probability of this event as $\alpha_{d}$

$$
\boldsymbol{P r}\left\{\mathbf{A}^{\mathbf{d}}\right\} \triangleq \alpha_{d}(N)
$$

Since all the events $\mathbf{A}_{i}$ are independent

$$
\alpha_{d}(N)=\left(1-e^{-N \mu}\right)^{d-1}
$$

The function $\alpha_{d}(N)$ is actually a distribution function since it shows the probability of event $\mathbf{A}^{\mathbf{d}}$ when at least $N$ packets are emitted by the attacker. The corresponding probability distribution can be approximated as a derivation of the function $\alpha_{d}$

$$
a_{d}=\frac{\partial \alpha_{d}}{\partial N}
$$

The function $a_{d}(N)$ is the probability distribution and it shows the probability that event $\mathbf{A}^{\mathbf{d}}$ occurs on the $N$ th packet.

The most interesting parameters of this function is the expected number of packets required to reconstruct the whole path and the upper bound (e.g. 90\%-bound) of packets required to reconstruct the whole path.

The expected number of packets generated by the attacker required to reconstruct the path from the victim back to attacker is given by the following:

$$
\mathbf{E}\left\{a_{d}\right\}=\sum_{N=1}^{\infty} N \cdot a_{d}(N)
$$

The approximation of this formula can be made by integration:

$$
\mathbf{E}\left\{a_{d}\right\} \approx \int_{0}^{\infty} N \cdot \mu(d-1) e^{-N \mu}\left(1-e^{-N \mu}\right)^{d-2} d N
$$

By using the identity

$$
\int_{0}^{\infty} N e^{-\mu s N} d N=\frac{1}{\mu^{2} s^{2}}
$$

yielding

$$
\begin{gathered}
\mathbf{E}\left\{a_{d}\right\} \approx \frac{d-1}{\mu} \sum_{i=1}^{d-1} \frac{(-1)^{i+1}}{i^{2}} \cdot\left(\begin{array}{c}
d-2 \\
i-1
\end{array}\right) \\
\mathbf{E}\left\{a_{d}\right\} \approx \frac{1}{\mu} \sum_{i=1}^{d-1} \frac{(-1)^{i+1}}{i} \cdot\left(\begin{array}{c}
d-1 \\
i
\end{array}\right)
\end{gathered}
$$

and finally

$$
\mathbf{E}\left\{a_{d}\right\} \approx \frac{1}{\mu} \sum_{i=1}^{d-1} \frac{1}{i}
$$

By using the approximation of the partial sum of harmonic series:

$$
\mathbf{E}\left\{a_{d}\right\} \approx \frac{\ln (d-1)+\gamma}{\mu},
$$

where $\gamma \approx 0.5772$ is the Euler's constant.

The second interesting parameter is $r$-bound of the number of packets required to complete the reconstruction of the path from the victim to the attacker. This parameter shows how many packets the attacker should issue to make sure that event $\mathbf{A}^{\mathbf{d}}$ is asserted with the probability $r$.

$$
\operatorname{Pr}\left\{\mathbf{A}^{\mathbf{d}}\right\}=\alpha_{d}(N) \geq r
$$

yielding

$$
N_{r} \geq-\frac{\ln \left(1-r^{\frac{1}{d-1}}\right)}{\mu}
$$

For the values of $r$ close to one this formula is simplified by the following approximation:

$$
N_{r} \geq \frac{\ln (d-1)-\ln (1-r)}{\mu}
$$

However, if the iTrace packets contain both the forward and the backward links, the reconstruction is still possible even if not all routers emits an iTrace packet. Consider routers $R_{i-1}, R_{i}$, and $R_{i+1}$, if routers $R_{i-1}$ and $R_{i+1}$ emit the iTrace packets with the forward and the backward 
links then the reconstruction of the path from $R_{i-1}$ to $R_{i+1}$ through $R_{i}$ is still possible.

Let event $\mathbf{B}_{i}$ be the event where an edge between routers $R_{i-1}$ and $R_{i}$ is sent to the victim either as a forward or as a backward link.

$$
\mathbf{B}_{i}=\mathbf{A}_{i-1} \cup \mathbf{A}_{i}
$$

It is possible to reconstruct the whole path from the victim to the attacker if the victim collects all the edges along the path. Let event $\mathbf{B}^{\mathbf{d}}$ be an event where all edges between the adjacent routers are collected at the victim. In general, it is not necessary that all the routers emits an iTrace packet.

$$
\mathbf{B}^{\mathbf{d}}=\bigcap_{i=1}^{d-1} \mathbf{B}_{i}
$$

Similar to the case with event $\mathbf{A}^{\mathbf{d}}$ we introduce a distribution function $\beta_{d}(N)$

$$
\operatorname{Pr}\left\{\mathbf{B}^{\mathbf{d}}\right\} \triangleq \beta_{d}(N),
$$

and distribution $b_{d}$

$$
b_{d}=\frac{\partial \beta_{d}}{\partial N}
$$

The calculations gives the following expression for the function $\beta_{d}(N)$ :

$$
\beta_{d}(N)=\sum_{i=1}^{d} D_{i}^{d-2}\left(1-e^{-\mu N}\right)^{d-i} \cdot e^{-\mu N(i-1)},
$$

where coefficients $D_{i}^{j}$ are found from the recurrent relation:

$$
D_{1}^{1}=D_{1}^{2}=D_{2}^{2}=1, D_{i}^{j}=D_{i}^{j-1}+D_{i-1}^{j-1}
$$

This equation, however, is too complicated to be used in practical calculations. Fortunately, function $\beta_{d}$ can be approximated by the function $\alpha_{d}$. The numerical analysis shows that the following approximation can be used in rough computations:

$$
\beta_{d}(N) \approx \alpha_{d}(k \cdot N),
$$

where $k \approx 1.3$ for the short paths and $k \approx 1.8$ for the long paths. In general, one can assume $k \approx 1.5$ for rough approximation.

This approximation allows avoiding the use of the complex function $\beta_{d}(N)$ in practical calculations. Particularly, for the expected number of packets:

$$
\mathbf{E}\left\{b_{d}\right\} \approx \frac{\mathbf{E}\left\{a_{d}\right\}}{k}
$$

and for upper bound:

$$
N_{r}^{(\beta)} \approx \frac{N_{r}^{(\alpha)}}{k}
$$

